\title{
MEMÓRIAS E REPRESENTAÇÕES SOCIAIS DO SISTEMA ÚNICO DE SAÚDE POR SEUS USUÁRIOS
}

\author{
Memories and social representations about the unified health system acording to \\ the users
}

Memorias y representaciones sociales del sistema único de salud por sus usuarios

Caren Camargo do Espírito Santo ${ }^{1}$

Thais Carvalho Gonçalves ${ }^{2}$

Denize Cristina de Oliveira ${ }^{3}$

Antonio Marcos Tosoli Gomes ${ }^{4}$

\section{RESUMO}

Esse estudo tem como objetivo analisar o conteúdo das memórias e das representações do Sistema Único de Saúde (SUS) desenvolvidas por seus usuários. Possui abordagem qualitativa, baseado na Teoria das Representações e Memória Sociais. Foram selecionadas 15 entrevistas de usuários da rede hospitalar e 15 da rede básica, e realizada análise de conteúdo temática. Originaram-se três categorias, porém este estudo refere-se apenas a uma categoria, denominada Representações Sociais e Memórias do SUS. Os sujeitos reconhecem o SUS como um sistema de saúde para todos. Sobressai o bom atendimento, assim como relatam as facilidades e dificuldades dos serviços de saúde. Relatam também a utilização dos serviços de saúde no setor privado e utilização do sistema de referência como um elemento facilitador. Conclui-se que a representação sobre o SUS é a universalidade e que este estudo permitiu entender como o usuário do SUS vivencia seu processo de implementação.

Palavras-chave: Sistema único de saúde. Política de saúde. Enfermagem em saúde pública.

\begin{abstract}
This study aims to analyze the content of the memories and representations of the Unified Health System (SUS), developed by its users. It has a qualitative approach, based on the Theory of Social Representations and Memory. We selected 15 interviews of users of the hospital network and 15 from basic network, and performed by a thematic content analysis. Originated three categories, but this study refers only to one category, called the Social Representations and Memories of the SUS. Subjects recognize the SUS as a system of health for all. Stands the proper care, reporting as well as the facilities and difficulties of health services. They also report the use of health services in the private sector and the use of referenced system as a facilitator. We conclude that the representation on the SUS is the universality and that this study allowed us to understand how the user experiences the SUS implementation process.
\end{abstract}

Keywords: Unified Health System. Health Policy. Public Health Nursing

\section{Resumen}

Este estudio tiene como objetivo analizar el contenido de las memorias y representaciones del Sistema Único de Salud (SUS) desarrolladas por sus usuarios. Es cualitativo, basado en la Teoría de las Representaciones y Memoria Sociales. Fueron seleccionadas 15 entrevistas de usuarios de la red de hospitales y 15 de la red básica, y se realizaron análisis de contenido temático. Originaron tres categorías, pero este estudio sólo se refiere a una, denominada Representaciones Sociales y Memorias del SUS. Los entrevistados reconocen a SUS como un sistema de salud para todos. Destacan el atendimiento adecuado, los puntos fuertes y las dificultades de los servicios de salud. Informan la utilización del sector privado de salud y del sistema de referencia como un facilitador. Concluye que la representación de SUS es la universalidad y que este estudio sirvió para comprender como el usuario vive su proceso de implementación.

Palabras-clave: Sistema Único de Salud. Política de Salud. Enfermería en Salud Pública

\footnotetext{
${ }^{1}$ Enfermeira. Mestranda do Programa de Pós-Graduação em Enfermagem da Universidade do Estado do Rio de Janeiro, Faculdade de Enfermagem. Rio de Janeiro-RJ. Brasil. E-mail: carencamargo.enf@gmail.com; ${ }^{2}$ Enfermeira. Residente em Hematologia e Hemoterapia pela Universidade Federal do Estado do Rio de Janeiro. Rio de Janeiro-RJ. Brasil. E-mail: athais_carvalho@hotmail.com.; ${ }^{3}$ Professora Titular da Faculdade de Enfermagem da Universidade do Estado do Rio de Janeiro (FENF/UERJ). Rio de Janeiro-RJ. Brasil. E-mail: dcouerj@gmail.com.; ${ }^{4}$ Professor Adjunto da FENF/UERJ. Doutor em Enfermagem pela Escola de Enfermagem Anna Nery/UFRJ (EEAN/UFRJ). Rio de Janeiro-RJ. Brasil. E-mail: mtosoli@gmail.com.
} 


\section{INTRODUÇÃO}

A Constituição Federal de $1988^{1}$ estabeleceu a saúde como um direito de todos e um dever do Estado, passando a ser entendida como um conjunto de determinantes, envolvendo as condições de moradia, transporte, trabalho e lazer, entre outros fatores. A partir dessa nova visão de saúde, foi criado 0 Sistema Único de Saúde (SUS), sendo este o atual sistema formado pelo conjunto de todas as ações e serviços de saúde prestado por órgãos e instituições públicas federais, estaduais e municipais, da administração direta e indireta e das fundações mantidas pelo Poder Público. ${ }^{2}$

Para entender o impacto do SUS e de seu acesso na população brasileira, a Pesquisa Nacional por Amostra em Domicílio (PNAD) feita pelo Instituto Brasileiro de Geografia e Estatística (IBGE) utilizou uma amostra de 384.834 pessoas no ano de 2003. Alguns dados que se relacionam com a cobertura do SUS no território nacional foram observados: dentre esses, mostram-se: média de consultas per capita no Brasil, de 2,4; 3,4 deram-se na infância e 4,0 em maiores de 64 anos; os postos e centros de saúde foram os serviços mais procurados, com uma taxa de $52 \%$, seguidos pelos consultórios particulares (18\%), ambulatórios hospitalares (17\%) e unidades de pronto-socorro (5,8\%); o número de consultas no Programa Saúde da Família (PSF) passou de 6.918 .985 para 72.834.885; e foram referidas cerca de sete internações por 100 habitantes, em um total de 12 milhões e 300 mil. $^{3}$

O SUS está embasado nos princípios doutrinários da universalidade, integralidade e equidade. A universalidade assegura o direito à saúde a todos os cidadãos. A integralidade visa levar em consideração as diversas dimensões do processo saúde-doença que afetam os indivíduos e as coletividades, de forma a percebê-los em sua totalidade, assegurando ações preventivas e, da mesma forma, curativas, conforme suas necessidades. 0 princípio da equidade aponta a necessidade de se reduzir as disparidades sociais e regionais existentes em nosso país, visando um maior equilíbrio. Há também outros princípios, de cunho organizativo, que visam dar racionalidade ao funcionamento do SUS sendo denominados da seguinte maneira: a regionalização e a hierarquização, a resolutividade, a descentralização e a participação da comunidade. . $^{4-5}$

0 grande número de utilização dos serviços públicos de saúde pela população brasileira, aliado a fatores como recursos humanos, financeiros, físicos, estrutura, organização e disponibilidade dos serviços e fatores biopsicossociais, influenciou, de forma maior ou menor, nas memórias e nas representações dos usuários, já que estas são multifacetadas, pois abarcam questões sociais, políticas, econômicas e culturais. Logo, o objetivo deste estudo é analisar o conteúdo das memórias e representações dos usuários do sistema da saúde acerca do SUS. Neste contexto, este estudo é pertinente, pois apresenta-se como indicador de como a implementação do atual sistema está sendo entendida e vivenciada, já que o SUS é uma política que funciona quando é colocada em prática pelas instituições, pelos usuários e pelos profissionais alocados nelas.

\section{METODOLOGIA}

Trata-se de um estudo de abordagem qualitativa baseado na Teoria das Representações Sociais e da Memória Social. A utilização da Memória Social como um elemento de referencial metodológico está fundamentada no tempo decorrido da implementação da nova política de saúde e na necessidade de visualizar esta implementação não somente sobre o prisma institucional, mas também através do olhar dos diversos atores sociais que aí interagem e, no caso deste estudo, dos usuários. ${ }^{6}$ Tratando-se do caráter e do grau de importância política e econômica do SUS é que a Teoria das Representações Sociais é justificada, sendo esta entendida como "uma forma de conhecimento socialmente elaborada e partilhada, tendo uma orientação prática e concorrendo para a construção de uma realidade comum a um conjunto social" 7:22

Os dados analisados no presente estudo fazem parte do Banco de Dados das pesquisas desenvolvidas sobre as representações e as memórias de profissionais e usuários da cidade do Rio de Janeiro sobre o Sistema Único de Saúde, de responsabilidade do projeto integrado "Políticas e Práticas de Saúde na Enfermagem: Comparações das Memórias e das Representações dos Usuários e Profissionais de Saúde". Para os fins deste trabalho, foram analisadas 30 entrevistas de usuários nos contextos de um hospital federal, geral e de grande porte, e de um centro municipal de saúde, sendo 15 entrevistas para cada cenário.

A seleção das entrevistas nestes diferentes cenários foi para identificar se há memórias e representações diferentes em cada contexto institucional. Tais instituições têm como características: existem há mais de 21 anos, de forma que construíram uma história dentro do sistema de saúde do município; têm história de prestação de serviços assistenciais anterior à implantação do SUS; e são de fácil acesso à população. Os sujeitos das entrevistas possuem as seguintes características: estão na faixa etária acima dos 40 anos e têm experiência como usuários do serviço anterior a 1988, ano da implantação do SUS. As entrevistas de cada instituição foram selecionadas aleatoriamente.

Em observância aos aspectos éticos e legais da resolução 196/96, do Conselho Nacional de Saúde, que normaliza a pesquisa com seres humanos, o projeto principal de pesquisa foi apresentado ao Comitê de Ética da UERJ, tendo sido aprovado, com protocolo n $n^{0}$. 006/2005. Antes de realizar as entrevistas foram apresentados, para cada entrevistado, os objetivos e metodologia do estudo, bem como o Termo de Consentimento Livre e Esclarecido e solicitado que os sujeitos 0 assinassem.

Para analisar os dados obtidos através das entrevistas, utilizou-se a análise de conteúdo temática-categorial. ${ }^{8-9}$ Neste sentido, as entrevistas foram divididas inicialmente em unidades 
de registro (UR), um determinado trecho discursivo que obedece a um tamanho previamente definido pelo pesquisador, que, neste caso, foi o tema; ou seja, o tamanho é delimitado em função da explicitação de uma temática, quer seja em uma palavra, uma frase ou um parágrafo. Para fins deste estudo, as URs recortadas referem-se apenas a memórias para as quais os sujeitos não relataram data, ou seja, foram selecionadas aquelas com as palavras "antes" e "antigamente" referindose ao SUS.

Foram elaboradas as categorias empíricas, considerando que são construídas a partir das falas dos sujeitos. Depois disso, foram submetidas à interpretação teórica. As categorias sintetizaram as UR e agregaram os significados existentes no texto em subconjuntos. Ao mesmo tempo, a elaboração das categorias obedeceu aos critérios de especificidade, em que uma mesma UR não foi classificada em duas categorias distintas, e comportou a maior parte do material analisado, traduzindo uma importância quantitativa em sua formulação.

\section{RESULTADOS E DISCUSSÕES}

Como já pontuado, foram analisadas 30 entrevistas, sendo 15 de um Hospital Federal e 15 de um Centro Municipal de Saúde, onde se verificaram 372 UR distribuídas em 48 temas que deram origem a três categorias, identificadas pelos números I a III: As representações sociais e memórias do INAMPS, Representações sociais e memórias do SUS e Aspectos profissionais, políticos e estruturais no processo de avaliação dos sistemas de saúde. Porém, trataremos aqui apenas da categoria II, Representações Sociais e Memórias do SUS, que representou $30,9 \%$ do total da análise, sendo composta de duas subcategorias, nomeadas de Aspectos avaliativos do SUS e Facilidades e dificuldades no contexto SUS.

\section{Subcategoria 1 - Aspectos Avaliativos do SUS}

Alguns usuários possuem uma representação positiva acerca do SUS, considerando o INAMPS como um sistema carente e com muitas dificuldades. A palavra "pior" é utilizada ao se comparar os dois sistemas. Ao expressar o INAMPS como um sistema "muito pior", percebe-se também que, apesar da positividade do SUS em relação ao INAMPS, os sujeitos se referem ao SUS como um sistema que também possui dificuldades.

\section{No tempo do INAMPS era muito pior. (E 32)}

Em relação à implantação do SUS e o reconhecimento dele como sistema de saúde posterior ao INAMPS, a maioria dos entrevistados não sabe dizer ao certo quando foi implantado. Há diferenças de datas entre as falas dos sujeitos, que varia de cinco a mais de vinte anos. Alguns citam datas a partir da primeira vez que 0 utilizaram, enquanto outros, a partir de quando escutaram falar.

Assim, a implantação do SUS é representada tanto como um processo perceptível no cotidiano dos usuários quanto como algo dissociado da realidade dos serviços e sem impacto no cotidiano deles..$^{10}$ Nota-se, portanto, que, à medida que aparece no contexto das memórias e das representações sociais dos usuários a falta da exatidão de quando o SUS foi implantado, seja por não utilizarem os seus serviços, ou por terem escutado falar, a sua implementação apresenta-se ainda como uma construção, repleta de contradições, que ainda está sendo percebida pelos usuários.

Os usuários perceberam que, após a implantação do SUS, todos os cidadãos passaram a ter o direito ao acesso aos serviços de saúde, trabalhadores e não trabalhadores, o que não acontecia no sistema anterior. Essa percepção é um princípio do novo sistema, denominado universalidade.

Eu lembro perfeitamente que qualquer pessoa de idade, sexo ou religião, trabalhando ou não, tem direito à assistência médica do SUS. (E 23)

0 princípio da universalidade é a oferta de serviços e ações de saúde a todos aqueles que deles necessitem, ou seja, deve ser assegurado o atendimento à saúde a todos os cidadãos que procurem por assistência. Este princípio está assegurado na Constituição Federal e constitui-se como um direito que deve ser garantido pelo Estado. ${ }^{5}$

Outro ponto destacado pelos sujeitos foi a participação social nos serviços do SUS. Alguns relatam a existência de reuniões dentro da unidade de saúde nas quais os usuários participavam ativamente, de diversas formas não exemplificadas. A referência à par ticipação social pelos entrevistados é positiva, pois, dentre os princípios do SUS, a participação ou o controle social na saúde, regulamentada pela Lei 8142/90, destaca-se como de grande importância, pois é a garantia de que a população participará do processo de formulação e de controle das políticas de saúde. ${ }^{11}$

No que tange ao atendimento nas unidades privadas, alguns o utilizavam por possuir plano de saúde da empresa onde trabalhavam, enquanto outros, apenas em uma única situação. Essas experiências geraram percepções distintas em relação ao atendimento nas clínicas particulares: uma boa e outra de mau atendimento.

Nunca frequentava o SUS. Tinha SEMIC, a firma que trabalhava que era multinacional, e os doentes eram atendidos pela SEMIC, mas não tem mais. Era atendido pela SEMIC, e o pessoal falava bem da SEMIC, lá é bom. (E 12) 
No particular só teve uma clínica que eu fiquei, mas mesmo assim não gostei. E não era pelo SUS, foi particular, fizeram uns exames e não descobriam que eu tinha diabetes, agora, não sei por quê. Igual a uma enfermeira que ia colocar o garrote no braço e descobriu logo o que eu tinha. (E 27)

Destaca-se que é definido, na Constituição Federal, que o setor privado tem a sua participação na saúde de forma complementar. Destaca-se, ainda, que este sistema misto resultou na situação atual, em que cerca de $20 \%$ da população brasileira tem, além do direito de acesso ao serviço público, a serviços de saúde providos por planos e seguros de saúde privados, uma vez que a rede pública tem dificuldades em atender, confortavelmente, os $80 \%$ da população que dependem exclusivamente do sistema público de saúde. ${ }^{12}$

Quanto aos atores sociais presentes nos cenários institucionais, além da figura do médico, aparece a do enfermeiro. Sendo assim, para alguns, a boa qualidade de atendimento também está relacionada ao tratamento dado aos usuários.

Sempre foi tudo certinho, direitinho, as enfermeiras me tratando muito bem. Eu não tive decepção com ninguém, graças a Deus. (E 05)

0 destaque do profissional enfermeiro pelos sujeitos pode ser embasado na afirmação que sendo o SUS resultado de lutas constantes das últimas décadas. A enfermagem brasileira, durante esse processo, tem participado com maior ou menor intensidade, dependendo do momento político e dos elementos que participam das entidades representativas da categoria. Esse profissional mostra-se bem ativo no processo de cuidado e assistência à saúde, sendo, inclusive, aquele que permanece maior tempo com os usuários e mais próximos destes. ${ }^{13}$

Além disso, o enfermeiro está inserido em todas as etapas do processo de trabalho em saúde: na administração, na organização, na coordenação, no acompanhamento, na tomada de decisão e na avaliação das ações desenvolvidas. Assim, cabe a ele conhecer, opinar, participar, decidir e intervir sobre as diretrizes sociais e as políticas no delineamento das ações, pois a dimensão política é estabelecida pela capacidade de mobilizar grupos sociais de acordo com as demandas, necessidades e interesses, inclusive dos usuários. ${ }^{14}$

Percebe-se que a confiabilidade nos serviços públicos de saúde foi adquirida quando os usuários passaram a confiar nos profissionais que 0 atenderam de alguma forma que os satisfizeram em suas necessidades.

E eu tinha uma necessidade imediata, fui para o particular e estou continuando o tratamento aqui no hospital, porque tive acesso aos grandes médicos, grandes pessoas que me atenderam. (E 23)

Na primeira vez foi bom, porque eu tinha esse problema que médico nenhum controlava a minha pressão, e tinha uma menina que fazia estágio de enfermagem aqui e me aconselhou a vir, eu vime fui muito bem atendida, e continuei meu tratamento aqui com a minha pressão controlada. (E 10)

Considera-se que a saúde se faz com pessoas e entre pessoas, com a mediação das tecnologias geradas pela ciência e pelo conhecimento popular. Trata-se de uma relação humana entre sujeitos, com suas potencialidades, limites e saberes. Quer seja na perspectiva da assistência para a recuperação ou a prevenção de agravo ou doença, quer seja para a promoção da saúde, essa produção se realiza na relação entre indivíduos e uma equipe de saúde, entre uma população e seu sistema público de saúde. ${ }^{15}$

Para alguns usuários, o fato de conseguir ser bem atendido em uma unidade pública de saúde é uma questão de sorte. Percebe-se que os usuários esperam que, a qualquer momento, o atendimento pode ser ruim, não causando, portanto, surpresa. Nota-se que, quando relatam suas experiências de um bom atendimento no SUS, muitos sujeitos utilizaram a expressão "graças a Deus", ligada à fé, que pode estar relacionada ao fato de que o bom atendimento não é o esperado quando se trata de um serviço público de saúde, e sim algo divino.

Dessa vez eu dei sorte, eu vim e consegui, é a segunda vez que eu venho aqui. É bom, até agora eu estou gostando. (E 25)

Eu, graças a Deus, quando precisei do SUS, fui bem atendida. (E 32)

Diante disso, percebe-se que o SUS é um sistema que possui falhas no cumprimento de suas leis, principalmente no que se refere à universalidade, devido à denominada universalização excludente, ao processo de hierarquização e à deficiência do funcionamento da rede básica como porta de entrada, lotando as emergências dos hospitais públicos. Mas, ainda assim, o atual sistema se mostra como positivo, principalmente na relação profissional-usuário, na participação social e na resolutividade.

\section{Subcategoria 2 - Facilidades e dificuldades no contexto do SUS}

Dentre os elementos facilitadores do SUS, o que ganha destaque é a facilidade do funcionamento do sistema de referência citado pela maioria, que étido como algo que funciona. 0 sucesso em conseguir atendimento por um encaminhamento, 
para os sujeitos, também está relacionada aos assistentes sociais dos hospitais, que possuem a imagem de facilitadores para o atendimento. 0 sistema de contrarreferência, por sua vez, não pode ser visualizado nas entrevistas.

Quando eu fui encaminhado pelo SUS, com aquele carimbo do SUS no papel, o atendimento foi melhor. (E 12)

Primeiro, eu comecei a me tratar em São João de Meriti. Depois, eu fui para o PAM [cita o nome do $P A M]$, ali na Cinelândia, e sempre fiquei assim. Depois me deram um encaminhamento para eu procurar endocrinologista. Então, eu corri os hospitais, fui lá no hospital [cita o nome do hospital], e não estavam aceitando, fui para o hospital [cita o nome de outro hospital], não estava aceitando, vim aqui e também não estava. Então, meu marido falou: Não, vamos falar com assistente social, graças a ela eu consegui. Ela conseguiu marcar para mim. (E 22)

0 fato de 0 sistema de contrarreferência não ser verbalizado e, portanto, de início, não ser percebido pelos entrevistados, demonstra uma das fragilidades de dois princípios do SUS: a integralidade e a hierarquização. A hierarquização diz respeito ao fluxo dos níveis de atenção: primário, secundário e terciário, os quais seguem à ordem dos níveis de complexidade. 0 sistema de referência e de contrarreferência é o caminho para 0 alcance da integralidade, que é entendida como a assistência às pessoas por intermédio de ações de promoção, proteção e recuperação da saúde com a realização integrada das ações assistenciais e das atividades preventivas, em todos os níveis de complexidade. ${ }^{2}$

Em contrapartida, a dificuldade em conseguir atendimento, sem encaminhamentos, também foi relatada, pela maioria dos usuários, por experiências vividas pelos próprios sujeitos e por informações adquiridas de outra forma que não seja a vivenciada. Os motivos apresentados para justificar a dificuldade de atendimento foram a desmarcação de consultas, as filas extensas, a demora para conseguir ser atendido e a falta de profissionais que acarretam na demora da marcação de consulta.

Antigamente não tinha médico, marcava três, quatro meses, até um ano para ser consultado, às vezes chegava no SUS para ser consultado, e diziam o médico não vinha. (E 35)

Uma cartilha desenvolvida pelo Ministério da Saúde ${ }^{16}$ enumera algumas dificuldades encontradas no SUS, como o fato de muitos não conseguirem acesso ao SUS, principalmente nos grandes centros, onde é longa a fila de espera para consultas, exames e cirurgias. Dependendo do local, é comum não haver vagas para internação, faltam médicos, pessoal, medicamentos e até insumos básicos. Também é grande a demora nos encaminhamentos e na marcação para serviços especializados.

A fila como condição para se obter atendimento em saúde apresentou-se como uma imagem destacada na percepção do senso comum quando se aborda a universalidade do acesso no âmbito do sistema. Colocou-se, portanto, como barreira a ser vencida para que 0 atendimento se concretize, na qual a espera (dimensão temporal), a demanda a ser resolvida (dimensão existencial) e a solução - ou não - do problema (dimensão resolutiva) se misturam na representação do próprio sistema, com resultados nem sempre garantidos. ${ }^{17}$

Em relação às facilidades apresentadas pelos sujeitos encontra-se a de realização de exames que, em sua maioria, são realizados dentro da unidade de saúde onde o usuário costuma ser atendido. Segundo eles, não há facilidade para um determinado tipo de exame, e sim para qualquer que seja solicitado. Destaca-se, também, a agilidade para a sua realização.

Na mesma hora ele fez os exames, não deu para ver, então fez uma tomografia computadorizada que identificou que era o intestino. (E 28)

Os entrevistados confirmam o acesso à realização de exames no SUS ao afirmarem que conseguem realizar todos oS tipos necessários ao seu atendimento. Neste contexto, os avanços tecnológicos e farmacológicos da atualidade vêm possibilitando o aumento das chances de sobrevivência e de melhoria da qualidade de vida para muitas pessoas. Neste contexto, diante da facilidade em obter medicação no SUS há a valorização do programa do governo Remédio em Casa, que apareceu apenas nas falas dos sujeitos que foram entrevistados no Centro Municipal de Saúde.

\section{A minha senhora entrou nesse programa de receber remédio em casa. (E 12)}

Para melhor entendimento desse projeto, Remédio em Casa é uma iniciativa da Prefeitura Municipal do Rio de Janeiro que entrega, em domićlio, medicamentos para algumas doenças crônico-degenerativas com o objetivo de otimizar o controle clínico e epidemiológico e contribuir com o gerenciamento e a avaliação das políticas públicas de saúde. Conforme entrevista em outro estudo, os usuários demonstramse satisfeitos ao projeto e depois que passaram a usufruí-lo o apoiam, revelando ser uma ação considerada séria e que veio para otimizar e aperfeiçoar a vida da população, permitindo uma melhor resolução no avanço do tratamento das doenças

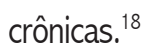

Ressalta-se que os sujeitos possuem uma percepção do sistema de saúde contraditória, em que se observa 
posicionamentos negativos e positivos. Esta tensão entre a negatividade e a positividade é fruto, além das dificuldades próprias do atendimento, de informações difundidas pela mídia - nem sempre disseminadoras dos fatos com a imparcialidade necessária -, de experiências com instituições e profissionais em situações de fragilidade e de representações negativas construídas coletivamente nas conversas cotidianas. ${ }^{10}$

Sendo assim, apesar das dificuldades para conseguir encaminhamentos dentro do SUS, ele é funcionante, tendo uma representação positiva para a maioria dos entrevistados. Isso também se reflete na obtenção do atendimento, que embora haja obstáculos, é concretizado. De certa forma, a realização dos exames também ocorre, quando são solicitados. Nota-se que o programa do governo no qual os usuários do SUS recebem seus remédios em casa foi um aspecto muito positivo e facilitador no cotidiano dos clientes, tendo uma representação positiva para a totalidade dos entrevistados.

\section{CONSIDERAÇÕES FINAIS}

Diante da análise desse trabalho, conclui-se que a dificuldade em lembrar quando o SUS foi implantado ou não sabê-lo gera um impacto negativo sobre os usuários, já que não lembrar pode significar não reconhecer que houve uma mudança de um sistema para outro. Isso demonstra que se não há percepção de mudança, pode não existir a percepção de direitos e de deveres, interferindo, portanto, diretamente no exercício da cidadania. Sendo os usuários participantes ativos nas políticas públicas e em movimentos sociais, inclusive nos que resultaram na implantação do SUS, torna-se necessário que incorporem seus direitos e deveres para que esse sistema seja aperfeiçoado a cada dia.

Apesar disso, a representação sobre o SUS dos que perceberam as diferenças entre os dois sistemas é a universalidade, que possui percepções distintas de positividade e negatividade, partindo-se de que os trabalhadores formais referem uma negatividade, enquanto os trabalhadores informais relatam positividade. Assim, esse princípio do SUS foi algo marcante que causou e ainda causa um impacto no cotidiano dos usuários. Esse impacto pode ter sido/ser a chance de conseguir ser atendido ou a saída da população com boas condições financeiras para o setor privado.

Vale destacar a ênfase no relato do sistema de referência e a não verbalização do sistema de contrarreferência pelos usuários, que sugere uma falha na execução de alguns princípios do SUS, destacando-se os de integralidade e de hierarquização. Esse fato interfere diretamente na qualidade de vida e na melhora das condições de saúde desses atores socais, na medida em que não se respeitam as complexidades demandadas a partir das necessidades do usuário. Torna-se evidente uma organização do sistema de referência e de contrarreferência para que os usuários sejam atendidos em sua integralidade e de forma humanizada.
A imagem negativa do atual sistema está nas filas extensas e na demora por atendimento, e o fato de conseguir atendimento está relacionado ao divino e à sorte, elementos abstratos, não mensuráveis e que não se pode controlar. Apesar disso, o SUS se mostra como positivo no cotidiano dos sujeitos até para os que utilizam o setor privado de saúde. Isso contribui para que a representação do SUS esteja na universalidade do acesso e demonstra que este é um princípio respeitado.

A qualidade da assistência não depende apenas da disponibilidade de recursos materiais, humanos e financeiros, mas sim da qualidade destes e principalmente de como 0 profissional de saúde conduz a assistência. A qualidade do profissional é um fator que persiste ao longo dos sistemas públicos de saúde e que deve ser levado em consideração, pois a postura profissional sai do âmbito de uma consulta e abarca questões de valorização, humanização e acolhimento do usuário, o que interfere diretamente na terapêutica, na adesão ao tratamento e, consequentemente, na promoção e na recuperação da saúde.

Destaca-se a importância do vínculo entre profissional e o usuário para a construção de saberes e a prestação da assistência de qualidade. Diante disso, o enfermeiro poderá, também, assumir o papel de propagador de conhecimento para os demais usuários, fortalecendo, assim, a participação social nas decisões políticas do sistema de saúde e estimulando os demais profissionais da saúde e os próprios usuários a experienciarem as mudanças que a incorporação desses princípios pode trazer para as demandas, principalmente dos usuários. Sendo assim, todos têm o direito de conhecer as diretrizes do atual sistema e o dever de colocá-las em prática para que o SUS adquira a forma de um sistema sólido em detrimento de um ainda em processo de implementação.

Em vista disso as memórias e as representações permitiram saber e entender como o usuário do SUS compreende e vivencia seu processo de implementação. Isso gera um grande impacto na vida profissional do enfermeiro, pois é neste contexto que se encontra a sua relevância. Seja ocupando cargos de gerência ou lidando diretamente com o usuário na assistência de enfermagem, esse profissional tem papel fundamental nas políticas públicas de saúde, coordenando programas ou fazendoos funcionarem através da sua prática assistencial, além de estarem sempre junto do usuário, conhecendo sua realidade. Aliado a isso, a enfermagem possui uma grande força de trabalho. Todos esses fatores levam-nos a perceber a posição de destaque do enfermeiro diante do funcionamento das políticas de saúde do SUS.

Portanto, o enfermeiro deve reconhecer que as memórias e as representações, sejam elas positivas ou negativas, acerca do atual sistema também foram influenciadas pelo seu comportamento e atitude. Ao mesmo tempo, deve aprofundar o seu potencial para a transformação e a construção de novas representações dos usuários acerca do SUS que possam condizer com o que está definido em lei. A reorganização 
das práticas de enfermagem fundamentadas nas diretrizes desse sistema pode ser um caminho para o alcance dessa transformação, e a avaliação dos serviços e das políticas de saúde pelos usuários através do acesso às suas memórias e suas representações pode ser um indicador, assim como foi neste estudo, do funcionamento do SUS.

\section{REFERÊNCIAS}

1- Constituição 1988 (BR). Constituição da República Federativa do Brasil, de 05 de outubro de 1988. Diário Oficial da República Federativa do Brasil. Brasília (DF): Senado; 1988. Título VIII. Da Ordem Social. Capítulo II. Seção II. Da Saúde. Arts 196-200.

2- Lei 8.080, de 19 de setembro de 1990. Dispõe sobre as condições para a promoção, proteção e recuperação da saúde, a organização e o funcionamento dos serviços correspondentes e dá outras providências. Diário Oficial da República Federativa do Brasil, Brasília (DF), 20 set 1990: Seção 1: 18.055.

3- Ministério da Saúde (BR). Painel de indicadores do SUS. Brasília (DF); 2006.

4-Vasconcelos C, Pasche D. 0 Sistema Único de Saúde. In: Campos GWS, Minayo MCS, Akerman M, Drumond Júnior M, Carvalho YM, organizadores. Tratado de Saúde Coletiva. São Paulo: Hucitec; 2006. p. 531-62.

5- Ministério da Saúde (BR). Sistema Único de Saúde- SUS: princípios e conquistas. Brasilia (DF); 2000.

6- Oliveira DC, Sá CP, Gomes AMT, Fernandez SA. 0 acesso ao sistema público de saúde analisado a partir das memórias e representações dos seus profissionais. Resumos dos trabalhos apresentados no $56^{\circ}$ Congresso Brasileiro de Enfermagem; 2004 out 24-29; Gramado, Brasil. Gramado : ABEn; 2004. p. 257.

7- Jodelet D. Representações sociais: um domínio em expansão. In: Jodelet D, organizadora. As representações sociais. Rio de Janeiro: Eduerj; 2001. p.17-44.

8- Bardin L. Análise de conteúdo. 5ª ed. Lisboa: Ed. 70; 2010.

9- Oliveira DC. Análise de conteúdo temático-categorial: uma proposta de sistematização. Rev Enferm. UERJ. 2008 out/dez; 16(4): 569-77.

10- Oliveira DC, Sá CP, Gomes AMT, Ramos RS, Pereira NA, Santos WCR. A política pública de saúde brasileira: representação e memória social de profissionais. Cad Saude Publica. 2000 jan; 24(1): 197-206.

11- Lei 8.142, de 28 de dezembro de 1990. Dispõe sobre a participação da comunidade na gestão do Sistema Único de Saúde - SUS e sobre as transferências intergovernamentais de recursos financeiros na área da saúde e dá outras providências. Diário Oficial da República Federativa do Brasil, Brasilia (DF), 31 dez 1990.
12- Fonseca AL. Portabilidade em planos de saúde no Brasil [dissertação]. Rio de Janeiro: Fundação Oswaldo Cruz, Escola Nacional de Saúde Pública,; 2004.

13- Melo MRA, Fávero N, Évora YDM, Nakao JRS. Modificações no atendimento de enfermagem hospitalar decorrentes do Sistema Único de Saúde -SUS. Rev Latino-am Enfermagem. 1998 out; 6(4): 5-14.

14- Persegona KR, Lenardt MH, Rocha DLB, Zagonel IPS. 0 conhecimento político na atuação do enfermeiro. Esc Anna Nery. 2009 jul/set; 13(3): 645-50.

15- Inojosa RM. Acolhimento: a qualificação do encontro entre profissionais de saúde e usuários. Anais do $10^{\circ}$ Congreso Internacional del CLAD sobre la Reforma del Estado y de la Administración Pública; 2005 out 18-21; Santiago, Chile. Santiago: CLAD; 2005. p.1-13.

16- Ministério da Saúde (BR). O SUS pode ser seu melhor plano de saúde. $2^{\mathrm{a}}$ ed. Brasília (DF); 2003.

17- Pontes APM, Cesso RGD, Oliveira DC, Gomes AMT. 0 princípio de universalidade do acesso aos serviços de saúde: o que pensam os usuários? Esc Anna Nery. 2009 jul/set; 13(3): 500-07.

18- Simões JM, Monteiro MG. Estratégias de apoio às políticas públicas de saúde: a experiência do Projeto Remédio em Casa. Mundo Sal de. 2006 jun; 30(2): 289-99. 